\title{
SUFFICIENT CONDITIONS THAT A PEANO-INTERIOR FUNCTION HAVE AN EXTENSION WITH CONSTANT BOUNDARY VALUES $\left({ }^{1}\right)$
}

\author{
BY \\ WILLIAM C. FOX
}

1. Introduction. The subject of Peano-interior functions was first introduced in [1]. Certain technical terms and facts (from [1]) will be reviewed in this paragraph for ease of reference. A Peano-interior function is a real function $f$, continuous on the 2-manifold, with (possibly empty) boundary, $M$, which is interior on the interior of $M$ and each level curve of which is locally connected at those of its points which are interior points of $M$. By I: 5.1 of [1] the last stated property may be replaced (with no loss of generality) by the condition that if $p$ is an interior point of $M$ then the level curve containing $p$ is, in some neighborhood of $p$, a finite linear graph of which $p$ is an interior point. The order of a point in a linear graph is the number of edges of which the point is an element except when the point is not a vertex, in which case its order is two. The multiplicity of an interior point of $M$ (relative to $f$ ) is given by $(k / 2)-1$ where $k$ is the order of the point in its level curve. (Necessarily, $k$ is even.) A critical point of $f$ is an interior point of $M$ whose multiplicity is positive. The Peano-interior function $f$ is canonical on $M$ if each boundary curve of $M$ is a level curve of $f$. If $V$ is any subset of $M$ and $x$ is a point of $M$ then $V^{x}$ denotes the set $\{p: p \in V, f(p)=f(x)\}$. Thus $V^{x}$ is that part of the level curve of $f$ containing $x$ which is also in $V$.

For canonical Peano-interior functions $f$ on compact orientable 2-manifolds with boundary the sum of the multiplicities of the critical points of $f$ is less, by one, than the first Betti number (with integral coefficients) of the manifold, according to $\mathrm{V}: 3.1$ of [1]. This fact is the starting point of the present investigation. The purpose of this paper is to evaluate the sum of the multiplicities of the critical points of Peano-interior functions with boundary values more general than those permitted by the restriction "canonical." The procedure to be followed may be described as follows:

First, one attaches "cuffs" to the boundary components of the manifold. Second, one extends the given Peano-interior function to be a canonical

Presented to the Society, December 29, 1953 under the title Critical points of real functions defined on two-manifolds; received by the editors September 3, 1956 and, in revised form, April $18,1957$.

(1) The preparation of this paper was supported in part by the Office of Ordnance Research, U. S. Army, Contract DA-19-020-ORD-3778. Except for $\$ 10$, it is based on the final chapter of the author's dissertation, submitted in partial fulfillment of the requirements for the degree of Doctor of Philosophy in the University of Michigan, July, 1954. 
Peano-interior function on some 2-manifold contained between the original one and that obtained by attaching the "cuffs." Third, one determines precisely what is the sum of the multiplicities of those critical points (of the new function) which are in the new manifold but are not in the interior of the original manifold. Finally one applies V:3.1 of [1] to the extended function which is now canonical on the new manifold. From the last two steps one can obtain a formula for the sum of the multiplicities of the critical points in the interior of the original manifold. Evidently the possibility of extending a Peano-interior function to be canonical is of some intrinsic interest. For this reason the extension will be discussed here in a more general setting than that warranted solely by the purpose of evaluating the sum of the multiplicities of the critical points in the given manifold.

2. Preliminaries. Let $M$ be a separable metric 2 -manifold with (a possibly empty) boundary. Then each nonempty boundary component (or boundary curve) of $M$ is a homeomorphic image of a simple closed curve (a circle) or of an interval of real numbers without any end points (open arc). In the first event one can attach (by suitable identifications) to the boundary curve a closed plane annulus. In the second event one can attach a closed infinite plane strip. One thus constructs a new manifold $C(M)$ which contains $M$, is homeomorphic with $M$, and such that each component of $C(M)-M$ is topologically an annulus or a strip (except for one "missing" edge still in $M$ ). These components are called cuffs, which constitutes the only reason for the use of " $C$ " in the symbol " $C(M)$."

So far as the extension problem is concerned, it is necessary only that the new manifold contain the interior of $M$, for the ultimate goal is to determine the sum of the multiplicities of critical points in the interior of $M$. Any manifold (with boundary) $M^{*}$ contained between the interior of $M$ and $C(M)$ is a possible candidate for the desired domain of canonical extension. As such, certain boundary points of $M$ will be the subject of special attention (relative to $M^{*}$ ). Each boundary point of $M$ which is the limit of a sequence of points in the boundary of $M^{*}$ no two of which are in the same boundary curve of $M^{*}$ is called a geometric singularity relative to $M^{*}$. (Evidently no geometric singularity relative to $M^{*}$ can be a point of $M^{*}$.) These points are called geometric singularities because failure to extend a given Peano-interior function $f$ in a neighborhood of one of them arises exclusively from the choice of $M^{*}$ and depends on the function $f$ only so far as the choice of $M^{*}$ depends on $f$.

With this terminology established, one can describe specifically some of the various properties to be required of the desired extension. If $G$ is any nonempty set in the boundary of the separable metric 2-manifold $M$ then a $G$-canonical extension of the Peano-interior function $f: M \rightarrow E^{1}$ is any canonical Peano-interior function $f^{*}: M^{*} \rightarrow E^{1}$ such that:

2a. The restriction of $f^{*}$ to $M \cap M^{*}$ coincides with $f$. 
2b. $M^{*}$ is contained between the interior of $M$ and $C(M), G$ is the set of geometric singularities relative to $M^{*}$ and $M-G \subset M^{*}$.

2c. Each boundary curve of $M^{*}$ which does not meet the boundary of $C(M)$ is compact; each boundary curve of $M^{*}$ which does meet the boundary of $C(M)$ is a subset of that boundary.

$2 \mathrm{~d}$. Each critical point of $f^{*}$ is either a critical point of $f$ (and thus is necessarily an interior point of $M$ ) or is a boundary point of $M$.

One significance of $2 \mathrm{~b}$ and $2 \mathrm{c}$ is that $M^{*}$ will be compact if and only if $G$ is empty and $M$ is compact. Of course, $2 \mathrm{~d}$ simply puts a restriction on the "new" critical points.

Two main steps remain before the extension process itself can be described. First a prescription must be given whereby a particular choice of $G$ is made to depend on the given function $f$. Second, a prescription must be given (in terms of $f$ ) for the number of boundary curves possessed by $M^{*}$ (so that V: 3.1 of [1] can be applied to $f^{*}$ ). The terminology and notation for these prescriptions will occupy the remainder of this section.

Let $J$ be a boundary curve of the separable metric 2-manifold $M$ on which is given the Peano-interior function $f$. A point $p$ of $J$ is said to be detached from the interior of $M$ by points of smaller $f$-value, or more briefly $S$-detached, provided there exists a set $U$, open in $M$, such that $U$ contains $p$ and such that if $x$ is a point of $U-J$ then $f(x)$ is smaller than (i.e., $<) f(p)$. Let $D(S, J)$ denote the set of all $S$-detached points in $J$.

Lemma 2.1. If $p$ is an $S$-detached point then there is a topological open interval $I$ in $J$ containing $p$ such that $f(p) \geqq f(x)$ for every point $x$ in $I$.

Lemma 2.2. The function $f: M \rightarrow E^{1}$ is constant on each component of $D(S, J)$.

The proofs of these lemmas are simple exercises in manipulating the above definitions.

The "isolated" components of the set $D(S, J)$ will be placed in one to one correspondence with those boundary curves of $M^{*}$ which do not meet the boundary of $C(M)$. Thus the set $G$ of geometric singularities relative to $M^{*}$ will appear as the set of $S$-geometric singularities defined as follows: a point $p$ in $J$ which is a limit of a sequence of points of $D(S, J)$ no two of which are in the same component of $D(S, J)$ is called an $S$-geometric singularity. The set of all $S$-geometric singularities in $J$ is denoted by $G(S, J)$.

3 . The half-plane. Since the desired extension will be carried out over each boundary curve separately, it is sufficient to describe the extension in "the neighborhood" of a single boundary curve. The case when that curve is not compact is treated in this section.

THEOREM 3.1. Let $M$ be the closure of a half-plane whose boundary, $J$, is the real axis and let $f: M \rightarrow E^{1}$ be a Peano-interior function. Suppose for each point 
$p$ of $J$ which is not an $S$-geometric singularity that the set consisting of $M^{p}$, together with those points $x$ of $J$ for which $f(x)>f(p)$, is locally connected at $p$. Then there exists a $G(S, J)$-canonical extension $f^{*}: M^{*} \rightarrow E^{1}$ of $f$ such that the number of boundary curves of $M^{*}$ which do not meet the boundary of $C(M)$ is equal to the number of components of the set $D(S, J)-G(S, J)$ and the number of boundary curves of $M^{*}$ which do meet the boundary of $C(M)$ is equal to the number of components of the set $J-G(S, J)$.

What follows is a description of a proof of 3.1. Details are omitted in those circumstances where their presence would tend to make the overall account unbearably long and involved. When $C(M)$ is of finite connectivity the function constructed in the proof will have other properties (not claimed in the theorem's statement) which will be used later.

It is no loss of generality to assume that $f$ is bounded and, for that matter positive because the application of a bounded real homeomorphism to the range of any Peano-interior function $f$ will not change the topological properties of $f$. Let $M$ be taken as the upper half-plane and let $C(M)$ be the set of points $(x, y)$ with $y \geqq c$ for some negative constant $c$. The components of the set $D(S, J)$ are of two kinds, degenerate and not. Of the former there may be uncountably many but there are at most countably many of the latter, and $f$ is constant on each. Let $\left\{C_{i}\right\}$ be an enumeration of the nondegenerate components of $D(S, J)$. Some $S$-geometric singularities may be obtainable as limits of sequences of points in the sets $C_{i}$ no two of which are in the same set $C_{i}$. Such will be called nondegenerate $S$-geometric singularities.

The first step in the construction of $M^{*}$ is the following: (1) There exists a pair-wise disjoint sequence $\left\{A_{i}\right\}$ of doubly connected domains in the lower-half-plane such that for each $i$ the closure of the interval $C_{i}$ is the intersection with $J$ of the outer boundary of $A_{i}$. For what follows it does not matter how this sequence is constructed but only that its members be, topologically, annuluses.

Let $M^{\prime}$ denote the set obtained by removing the nondegenerate $S$-geometric singularities from the union of $M$ with the closures of the sets $A_{i}$. (If the sequence $\left\{C_{i}\right\}$ is infinite, this removal may be necessary to make certain that $M^{\prime}$ is a 2 -manifold.) One checks, as follows, that $M^{\prime}$ has only nondegenerate boundary curves. The boundary components of $M^{\prime}$ are of two sorts. First are the inner boundaries of the domains $A_{i}$. Second are the components formed by replacing each of the intervals $C_{i}$ with its complementary arc in the outer boundary of $A_{i}$ and then removing the set of nondegenerate $S$-geometric singularities. Each component of the second sort is necessarily nondegenerate.

The first step in the construction of $f^{*}$ is the following: (2) There exists a Peano-interior function $f^{\prime}: M^{\prime} \rightarrow E^{1}$ (2a) which coincides with $f$ on $M \cap M^{\prime}$, (2b) for which every component of $S$-detached points is degenerate. and 
(2c) which is canonical upon being restricted to each of the domains $A_{i}$ with the larger boundary value taken on the inner boundary curve.

To check (2) one notes that for each $i$ one can set up a topological map between the closure of $A_{i}$ and some geometric annulus centered at the origin such that inner boundaries correspond to outer boundaries. If one chooses the inner radius of the geometric annulus to equal the (constant) value of $f$ on $C_{i}$, then the absolute value of the map from $A_{i}$ to the geometric annulus serves to define a suitable $f^{\prime}$ on $A_{i}$. That the resulting $f^{\prime}: M^{\prime} \rightarrow E^{1}$ is Peanointerior may be checked by using the characterization of $\mathrm{I}: 5.1$ in [1] described in the first paragraph of this paper.

If the theorem (3.1) now being proved were known to be true under the additional hypothesis that $D(S, J)$ has no nondegenerate components (see $2 \mathrm{~b}$ ) then that fact could now be applied in a neighborhood of each boundary component of $M^{\prime}$ (except those in the closures of the sets $A_{i}$ ) to complete the proof (of 3.1). Therefore, it is now sufficient to give here a proof of 3.1 under this extra hypothesis (that $D(S, J)$ has no nondegenerate components).

One starts by observing that although $D(S, J)$ may not be countable, the set $D(S, J)-G(S, J)$ is countable, for if $p$ is a point of that set then there exists an open interval in $J$ containing $p$ which meets only finitely many components of $D(S, J)$. But under the new hypothesis, such components are points. Therefore there exists an interval in $J$ whose intersection with $D(S, J)$ is precisely the point $p$.

Let $\left\{p_{i}\right\}$ be an enumeration of the points in $D(S, J)-G(S, J)$. The next step in the construction of $M^{*}$ is the following: (3) There exists a pair-wise disjoint sequence $\left\{R_{i}\right\}$ of open topological annuluses in the lower half plane such that the closure of $R_{i}$ meets $J$ in the single point $p_{i}$. As in (2) there exists a continuous extension of $f$ to the union, $D$, of $M$ with the closures of the domains $R_{i}$ such that property (2c) holds on each $R_{i}$. (Of course, the set $D$ is not yet a 2 -manifold.)

The desired set $M^{*}$ is now defined to be $C(M)$ with the removal of the $S$-geometric singularities $G(S, J)$ together with those simply connected domains whose boundaries are the inner boundaries of the annuluses $R_{i}$. It remains to extend $f$ to all of $M^{*}$ from $D$. The extension will be constructed across each component of $J-G(S, J)$ separately. What follows is a description of the case in which $G(S, J)$ is empty but it serves equally well for any component of $J-G(S, J)$.

Let $E$ denote the union of $J$ with the outer boundaries of the annuluses $R_{i}$ and let $T$ denote a plane distinct from the one containing the above sets. (4) There exists a topological open interval $\epsilon$ separating the plane $T$ and a local homeomorphism $g$ of $\epsilon$ onto $E$ such that (4a) each point of $E$ has exactly one pre-image in $\epsilon$ except for the points $p_{i}$ each of which is covered by $g$ exactly twice, (4b) the $y$-coordinate (in $T$ ) of each point $z$ in $\epsilon$ measures the $f$-value of its $g$-image, i.e., $f(g(z))=\pi(z)$ (where $\pi$ is defined to be the projec- 
tion into the $y$-axis). Moreover, there exists a continuous extension of $g$ to the lower complementary domain $\delta$ (in $T$ ) of $\epsilon$ such that (4c) $g$ is $1-1$ on $\delta$ and (4d) $g$ maps some horizontal straight line in $\delta$ onto the lower boundary line $(y=c)$ of $C(M)$. Thus $g$ is a homeomorphism of $\delta$ onto the domain "below" $E$ and "above" the line $y=c$, although $E \cup g(\delta)$ is not homeomorphic with $\epsilon \cup \delta$. Now, let $\pi\left(g^{-1}\right)$ define $f^{*}$ on the closure of $g(\delta)$. Although $g^{-1}$ is not single valued on $E, \pi\left(g^{-1}\right)$ is single valued there. Certainly $f^{*}$ agrees with $f$ on $E$ and is continuous on $M^{*}$. Indeed $f^{*}$ is a canonical Peano-interior function on $g(\delta)$.

The interiority of $f^{*}$ on $M^{*}$ may be checked by showing that if $p$ is an interior point of $M^{*}$ then the $f^{*}$-image of every neighborhood of $p$ contains an open interval containing $f^{*}(p)$. By construction, the truth of this assertion is immediate everywhere except when $p$ is in $E$. If $p$ is an interior point of an interval in $J-\cup_{i} \bar{R}_{i}$ then necessarily $p$ is not in $D(S, J)$ so every neighborhood of $p$ contains points (in $M$ ) whose $f^{*}$-values are greater than $f^{*}(p)$. But by construction of $f^{*}$ (through $\pi\left(g^{-1}\right)$ ) every neighborhood of $p$ also contains points (in $M^{*}-M$ ) whose $f^{*}$-values are less than $f^{*}(p)$. Since $f^{*}$ is interior on each component of $M^{*}-E$, it follows that $f^{*}$ does have the desired property at $p$. If $p$ is a point of $E-J$, a similar argument applies. If $p$ is one of the points $p_{i}$ then the points in $R_{i}$ near $p_{i}$ bear larger $f^{*}$-values, those near $p_{i}$ in $M$ bear smaller values, since $p_{i}$ is $S$-detached. Otherwise, the same argument applies to this case also and there are no other cases.

It remains to show that if $p$ is an interior point of $M^{*}$ then $M^{* p}$ is locally connected at $p$. This proposition is evidently true unless $p$ is a point of $E$. If $p$ is an interior point of an interval in $J-U_{i} \bar{R}_{i}$ (whence $p$ is not in $D(S, J)$ ) then according to a hypothesis of this theorem (3.1) the set consisting of $M \cap M^{* p}$ together with those points of $J$ at which $f(x)>f(p)$ is locally connected at $p$. (In what follows, it will be assumed that $g$ has been used to "identify" points in $E$ with points in $\epsilon$.) Each component, $I(p)$ (necessarily nondegenerate) of the set $\{x: x \in J, f(x)>f(p)\}$ has common endpoints with at least one (circular) arc $I^{*}(p)$ in $M^{* p}-M$ by construction of the extension $f^{*}$ through $\pi\left(g^{-1}\right)$. If two different arcs $I^{*}(p)$ share endpoints with $I(p)$ then these two determine a circle, and that one is chosen whose radius vector sweeps through the same central angle as is needed to traverse $I(p)$. With all such choices made, there is a 1-1 correspondence between the sets $I(p)$ and $I^{*}(p)$. (Note: each set $I(p)$ is determined by its endpoints so that different sets $I(p)$ determine different sets $I^{*}(p)$.) Moreover, the sets $I^{*}(p)$ are pairwise disjoint (except possibly for endpoints) for if not then the circular arc determined by two of them which meet contains two pairs of endpoints of two arcs of the form $I(p)$, these endpoints being arranged on the circular arc in such a way that the corresponding sets $I(p)$ must meet, an impossibility. It is now a simple matter to choose a homeomorphism of $I(p)$ onto $I^{*}(p)$ which leaves their common endpoints point-wise fixed. Let each point of 
$M^{* p}$ be transformed by such a homeomorphism should the point be in one of the sets $I(p)$ and let the point be transformed into itself otherwise. This defines a homeomorphism of $M^{* p}$ onto the set $M^{p} \cup\{x: x \in J, f(x)>f(p)\}$. Since the latter is locally connected at $p$ by hypothesis, the former is also locally connected at $p$. The final case is that in which $p$ is one of the points $p_{i}$. Here the two cases discussed above are both present and the argument is modified accordingly. In this way one verifies that $f^{*}$ is a (canonical) Peanointerior function on $M^{*}$.

This procedure places all the nondegenerate components of $D(S, J)$ and those of the degenerate components of $D(S, J)$ which are not in $G(S, J)$ in a 1-1 correspondence with the boundary curves of $M^{*}$ which are interior to $C(M)$. Also the components of $J-G(S, J)$ are placed in 1-1 correspondence with the set of boundary curves of $M^{*}$ which do meet (and hence are necessarily contained in) the boundary of $C(M)$. This finishes the description of the proof of 3.1 .

4. The exterior of the unit circle.

THEOREM 4.1. Theorem 3.1 remains true when $M$ is the closure of the exterior of the unit circle and $J$ is the unit circle itself.

The argument given for 3.1 remains valid word for word up to the construction of the set $\epsilon$. If, at that stage, $J$ is still compact (i.e., if there are no $S$-geometric singularities) then $\epsilon$ must be a simple closed curve: (4b) should read that $f(g(z))=|z|^{-1}$ for each $z$ in $\epsilon$, and, after "lower" is replaced by "exterior," (4d) should read that $g$ maps a circle centered about the origin in $\delta$ onto the boundary of $C(M)$. Finally, $f^{*}$ is defined by the rule $\left|g^{-1}\right|^{-1}$. With these changes, the proof of 3.1 applies also to 4.1 .

5. The dual extension. In the previous two sections, the letter " $S$ " has been used to distinguish the extensions constructed there from the dual extensions to be described here. If the inequalities which appear in the definition of an $S$-detached point are reversed, one has the definition of an $L$ detached point (a point which is detached from the interior of $M$ by points bearing larger $f$-values). Similarly one has the sets $D(L, J)$ and $G(L, J)$. Moreover, Lemma 2.2 remains true with " $S$ " replaced by " $L$."

THEOREM 5.1. Theorem 3.1 and Theorem 4.1 remain true if the inequality $f(x)>f(p)$ is reversed and if the letter " $S$ " is replaced throughout with the letter "L."

Two main changes are required in the proof of 3.1. First $M$ must be taken to be the lower-half-plane and $C(M)$ must be bounded above (instead of below) by a horizontal line. These changes entail some changes of language throughout, e.g., "lower" becomes "upper," etc. Second, the extensions of $f$ to the annuluses $A_{i}$ and $R_{i}$ must be made so that the values on the inner boundaries are smaller than those on the outer boundary. This change must 
also take place in the proof of 4.1 . The only other change needed in 4.1 is that $f$ is extended by the rule $\left|g^{-1}\right|$ instead of by $\left|g^{-1}\right|-1$.

Briefly put, the $S$-extensions are carried out, in the large, toward smaller values and their dual $L$-extensions are carried out, in the large, toward larger values.

6. Some refinements. In general, it would be desirable to have the new manifold $M^{*}$ be of minimal first Betti number. Since the genus of $M^{*}$ and that of $M$ are equal, the only feature of $M^{*}$ on which some control may be exercised is the number of its boundary curves. One can reduce the number of boundary curves of $M^{*}$ by reducing the number of components of the set $D(S, J)-G(S, J)$. All that is required (by the $S$-extension process) of these sets is that they contain no points of $D(L, J)$ and that they be intervals of constancy for $f$. One can for example, instead of the nondegenerate components of $D(S, J)$, deal with (in the start of the proof of 3.1) the components of the union of the closures of them, and thereby reduce the first Betti number of $M^{*}$. It may be desirable to repeat this process (of taking unions of closures of components) a finite number of times. (It is even possible to change the number of boundary curves of $M^{*}$ from being infinite to being finite.) One is then led to look at the nondegenerate components of the closure of $D(S, J)$ for these sets will contain all the components obtained by the finite repetition mentioned above. However, it would be essential to know that every Peano-interior function is constant on each component of the closure of $D(S, J)$. This constitutes an unsolved problem.

Of course, these remarks apply equally well to the dual $L$-extension.

7. The general case. A boundary curve $J$ of the 2 -manifold $M$ is said to be $S$-regular relative to the Peano-interior function $f: M \rightarrow E^{1}$ provided that for each point $p$ in $J$ the union of $M^{p}$ with the set $\{x: x \in J, f(x)>f(p)\}$ is locally connected at $p$. The curve $J$ is said to be $S$-free if it contains no $S$ geometric singularities. Dually, one defines the properties $L$-regular and $L$ free reversing the inequalities to be $f(x)<f(p)$.

THEOREM 7.1. Let $M$ be a separable metric 2-manifold with boundary consisting of the distinct components $J_{1}, \cdots, J_{n}$ and let $f: M \rightarrow E^{1}$ be Peanointerior. Suppose that $\delta$ is a function, with two values, defined on the integers from 1 to $n$ such that if $\delta(i)=S(=L)$ then $J$ is $S$ - (L-) regular. Then there exists a $\left(\cup_{i} G\left(\delta(i), J_{i}\right)\right)$-canonical extension $f^{*}: M^{*} \rightarrow E^{1}$ of $f$ such that the number of boundary curves of $M^{*}$ which do not meet the boundary of $C(M)$ is equal to the number of components of the set $\bigcup_{i}\left(D\left(\delta(i), J_{i}\right)-G\left(\delta(i), J_{i}\right)\right)$ and the number of those which meet the boundary of $C(M)$ is equal to the number of components of the set $\cup_{i}\left(J_{i}-G\left(\delta(i), J_{i}\right)\right)$.

This statement is simply a summary of the information in $3.1,4.1$, and 5.1.

8. The compact case. The extension described in 7.1 was developed orig- 
inally for its applications to the problem of counting the multiplicities of the critical points of a given Peano-interior function. For that purpose the following is a main result.

Theorem 8.1. Suppose the hypotheses of 7.1 are augmented by the assumption that if $\delta(i)=S(=L)$ then $J_{i}$ is $S-(L-)$ free and that $M$ is compact and orientable with genus $g$. Then $M^{*}$ is compact and orientable and the number, $n^{*}$, of boundary curves of $M^{*}$ is given by the sum of $n$ with the number of components of the set $U_{i} D\left(\delta(i), J_{i}\right)$. Moreover, the sum of the multiplicities of the critical points of $f^{*}$ in $M^{*}$ is $2 g+n^{*}-2$.

Of course, 8.1 is a corollary of 7.1 except for the last sentence which follows from $V: 3.1$ of [1].

The sum of the multiplicities of the critical points of $f$ in the interior of $M$ can now be evaluated provided the sum of the multiplicities of those critical points of $f^{*}$ which are located in the boundary of $M$ is known. This latter sum can be evaluated in the rather special situation described by the following lemma. (It would be of interest to devise a scheme for the evaluation of this sum more general than that described below.)

Lemma 8.2. Suppose $M$ is a separable metric 2-manifold, $J$ is a boundary curve of $M$, and $f: M \rightarrow E^{1}$ is Peano-interior. If each point of $J$ is $f$-normal, then $J$ is both $S$ - and L-regular relative to $f$.

In I: 5.1 of [1] it is shown that $f$-normality at a boundary point $p$ implies that, in a neighborhood of $p, M^{p}$ is a finite linear graph whose intersection with $J$ is an arc of $M^{p}$ or just $p$ itself, so that 8.2 is thereby proved. Moreover, under the condition of $f$-normality it makes sense to speak of the order of the boundary point $p$ in $M^{p}$. Suppose now that the conditions of 8.1 hold and that each boundary curve $J_{i}$ is $f$-normal. The $S$-increase in order of a point $p$ in $J_{i}$ is the difference between the order of $p$ in $M^{* p}$ and in $M^{p}$ when $f^{*}$ is the $S$-extension across $J_{i}$ constructed in the proof of 3.1. From that construction, one obtains the following conclusions provided the suppositions above remain in force.

(a) The $S$-increase in order of a point $p$ in $D\left(L, J_{i}\right)$ is zero, one, or two according as its order in $M^{p} \cap J_{i}$ is two, one, or zero.

(b) The $S$-increase in order of a point $p$ in $J_{i}$ but in neither $D\left(S, J_{i}\right)$ nor in $D\left(L, J_{i}\right)$ is either zero, one or two according as $p$ is an endpoint of two, one, or no nondegenerate components of $D\left(S, J_{i}\right)$.

(c) The $S$-increase in order of a point $p$ in $D\left(S, J_{i}\right)$ is either zero, one or two according as it is an interior point of a nondegenerate component of $D\left(S, J_{i}\right)$, an endpoint of a (and, necessarily, of exactly one) nondegenerate component of $D\left(S, J_{i}\right)$ or a degenerate component of $D\left(S, J_{i}\right)$.

Thus one can assign to each point $p$ of $J_{i}$ its $S$-index, a number equal to the order of $p$ in $M^{* p}$ (when $f^{*}$ is the $S$-extension across $J_{i}$ given by the 
proof of 3.1) in a way which requires knowledge only of the sets $M^{p}$ and $D\left(S, J_{i}\right)$. From the $S$-index $k$ one obtains the $S$-multiplicity by the rule $(k / 2)-1$. Dually, one has an $L$-index and an $L$-mutiplicity defined for each point in $J_{i}$. These facts may be combined with 8.1 and 8.2 to yield the following.

Theorem 8.3. Suppose that $M$ is a compact orientable 2-manifold of genus $g$ whose boundary consists of the distinct components $J_{1}, \cdots, J_{n}$, each of which is $f$-normal relative to the Peano-interior function $f: M \rightarrow E^{1}$. Suppose that $\delta$ is any function, with the values $S$ and $L$, defined on the integers from 1 to $n$. Then not only is each $J_{i}$ both $S$ - and L-regular and $S$ - and $L$-free, but also the sum of the multiplicities of the critical points of $f$ in $M$ is given by $\left(2 g+n^{*}-2\right)-m$ where $n^{*}$ is the number of components of the set $\bigcup_{i} D\left(\delta(i), J_{i}\right)$ and $m$ is the sum of the $\delta(i)$-multiplicities of the points in $J_{i}$ for $i=1, \cdots, n$.

The statement of 8.3 is intended to convey how the desired sum can be computed directly from knowledge of the sets $D\left(S, J_{i}\right), D\left(L, J_{i}\right)$ and of the $S$ - and $L$-multiplicities relative to $f$ of the points in the sets $J_{i}$. Of course one could pick out those points in $J_{i}$ of $\delta(i)$-multiplicity $>0$ and label them "critical boundary points." But in the event $J_{i}$ is both $S$ - and $L$-free (as is the case in 8.3) another set presents itself with equal force for the name "critical boundary" set. In [2, p. 14] Marston Morse defines certain boundary points to be critical points. Later, he introduces a multiplicity for these critical boundary points and he uses the sum of these multiplicities to measure (in effect) the difference between the sum of the multiplicities of the interior critical points and the first Betti number of the domain. In the context of his work there is but one way to measure this quantity and certain boundary points are involved with a role analogous to that of interior critical point, so it is reasonable to call them "critical boundary points." In the present context, such a nomenclature is no longer natural. Indeed, it may be (see §11) that a certain boundary point is a critical interior point under one extension and is a noncritical interior point under another extension.

9. Pseudo-harmonic extensions. It is natural in view of the fact (see $\mathrm{I}: 1$ in [1]) that every pseudo-harmonic function is necessarily Peano-interior to inquire whether the extension $f^{*}$ in the statement of 7.1 must be pseudoharmonic whenever $f$ is pseudo-harmonic. From the proof of 3.1 one sees that $f^{*}$ is certainly pseudo-harmonic at each interior point of $M^{*}$ except possibly at boundary points of $M$ which have remained as interior points of $M^{*}$. If one keeps in mind Tôki's characterization [3] of pseudo-harmonic functions (see I: 1 in [1]) one can guess how to strengthen the conditions of $S$-regularity and $L$-regularity to guarantee that $f^{*}$ will be pseudo-harmonic. If $J$ is a boundary curve of the metric 2-manifold $M$, and if $f: M \rightarrow E^{1}$ is Peano-interior, then $J$ is uniformly $S$-regular relative to $f$ provided first that $J$ is $f$-normal and second that at each point $p$ of $J$ (except for a discon- 
tinuum) and each $M$-neighborhood $U$ of $p$, there is an $M$-neighborhood $V$ of $p$ for which $\bar{V} \subset U$ and such that whenever $y$ is a point of $V$ the union of the set $V^{y}$ with the set $\{x: x \in J, f(x)>f(y)\} \cap V$ is entirely contained in some one component of the union of $U^{y}$ with $\{x: x \in J, f(x)>f(y)\} \cap U$. When the analysis carried out in the proof of 3.1 is applied and the components of $\{x: x \in J, f(x)>f(y)\}$ are replaced with arcs in $M^{*}-M$ on which $f=f(y)$ (to yield $M^{* y}$ from $M^{y} \cap\{x: x \in J, f(x)>f(y)\}$ ) one can prove that the regularity of $f^{*}$ on $M^{*}-M$ is sufficient to imply that Tôki's conditions for pseudoharmonicity of $f^{*}$ are indeed satisfied. Dually, one defines the property of being uniformly L-regular with analogous conclusions.

THEOREM 9.1. If the hypotheses of 7.1 are augmented by the assumptions that, for each $i, J_{i}$ is uniformly $\delta(i)$-regular and that $f$ is pseudo-harmonic (on the interior of $M$ ) then $f^{*}$ is necessarily pseudo-harmonic (on the interior of $M^{*}$ ).

10. Harmonic extensions, an unsolved problem. Suppose that $M$ is a Riemann surface with analytic boundary and that $f$ is harmonic on $M$ and satisfies the hypotheses of Theorem 9.1. Then $f$ admits a pseudo-harmonic extension $f^{*}$ to $M^{*}$. Question $\mathrm{I}$ is: Does there exist an analytic coordinate system $C^{*}$ for $M^{*}$ such that (i) the identity function sends $M$ conformally into $M^{*}$ and (ii) $f^{*}$ is harmonic on $M^{*}$ relative to $C^{*}$ ?

More generally, let $M$ be a domain in the orientable separable metric twomanifold $M^{*}$, let $C$ be an analytic coordinate system for $M$ and let $f$ be harmonic on $M$ relative to $C$. Question II is: If $f$ admits a pseudo-harmonic extension $f^{*}$ to $M^{*}$ does there exist an analytic coordinate system $C^{*}$ for $M^{*}$ with properties (i) and (ii)?

(Throughout, an analytic coordinate system of $X$ is taken to mean a family of homeomorphisms of plane domains onto open sets of $X$ whose ranges cover $X$ and are so related on the overlap that one local coordinate system followed by the other's inverse is always analytic.)

There are two facts (examples given at the end of the section) which make it more difficult than one might expect to answer these questions. They are: (a) It is possible for two $C^{*}$ 's to exist which have property (i), but which determine Riemann surfaces that are not conformally equivalent though they are homeomorphic; (b) It is possible for a single $f^{*}$ to be harmonic relative to two different $C^{*}$ 's which are not compatible, i.e., which contain members that are not analytically related on the overlap.

These facts should increase one's estimate of the probability of either question being answered affirmatively. However, even if it were known (III) that every pseudo-harmonic function is harmonic relative to some analytic coordinate system one still could not infer an affirmative answer to I or II because this coordinate system might not be compatible with the given $C$. (Tôki and Tarumoto [4] have announced that every pseudo-harmonic function is harmonic relative to some coordinate system but as Kaplan [5] points 
out they do not show that their coordinate system is analytic.) Of course, the reason III is hard to prove is that the local coordinate systems provided by the definition of pseudo-harmonicity (a pseudo-harmonic function is, locally, a harmonic function upon introduction of a suitable homeomorphism of a disk onto the locality in question) may be members of various analytic but noncompatible coordinate systems, as is illustrated by (b).

Finally, even if II were known to be answered "no," it could still be the case that $I$ has an affirmative answer by virtue of the special regularity conditions imposed by the hypotheses of 9.1 .

Example (a). Let $M$ be the open unit disk, let $C$ be the set whose single element is the identity function of $M$ onto $M$, and let $M^{*}$ be the plane. One constructs the homeomorphism $c^{*}$, on $M^{*}$, to be the identity function on $\bar{M}$ but to act on each ray $\left\{r e^{i \theta}: 1 \leqq r, \theta=\right.$ constant $\}$ in $M^{*}-M$ as a linear compression, sending it onto the interval $\left\{r e^{i \theta}: 1 \leqq r<2, \theta=\right.$ constant $\}$. If $C^{*}$ is the set whose single element is $c^{*-1}$ then a Riemann surface $\left(M^{*}, C^{*}\right)$ is determined which is conformally equivalent with a disk under the function $c^{*}$. Then $C^{*}$ shares property (i) with the coordinate system which consists of the identity function of $M^{*}$ onto $M^{*}$.

EXAmple (b). Let $g$ be any homeomorphism of the plane, $M^{*}$, which leaves vertical lines set-wise fixed. If $\pi$ denotes the projection of $(x, y)$ into the real axis, then $\pi g(x, y)=\pi(x, y)$. Then both $\pi$ and $\pi(g)$ are harmonic, although $g$ may certainly be nonanalytic. Thus $\pi$ is harmonic relative to two noncompatible coordinate systems for the plane, one containing the identity function and the other $\left(C^{*}\right)$ containing $g$. I do not know if $g$ can be chosen so that $\left(M^{*}, C^{*}\right)$ is conformally equivalent with a disk.

11. An example. One suggestion originating in the extension Theorem 7.1 is that a boundary point may be a critical point under one extension and a noncritical point under another extension. An explicit example of such a phenomenon will be given here to make this theoretical possibility more palatable. This (un-extended) example also has the property that it fails to be either $S$ - or $L$-regular whereas the extension found for the example indicates how one might formulate a general procedure which would not require the hypotheses of $S$-regularity and $L$-regularity. This procedure would be quite difficult to describe in any generality; perhaps the point of diminishing returns has been reached.

Let $A$ denote the closure of the plane domain bounded by the three circles $C_{1}, C_{2}$, and $C_{3}$, where the latter is the exterior boundary of $A$. Let $f: A \rightarrow E^{1}$ be the harmonic function which takes the value one on $C_{1}$ and $C_{2}$ and takes the value zero on $C_{3}$. Then, by III : 4.5 in [1], $f$ will have exactly one critical point, $p$, and its multiplicity is one. It is no loss of generality to suppose that $f(p)=1 / 2$ and to consider $f$ as a pseudo-harmonic function. The level curve pattern of $f$ is indicated in Figure 1.

Let the level curve containing $p$ be denoted by $K$, and let two points, $x_{1}$ and $y_{1}$, be chosen on one of the spokes of $K$. Let $X$ and $Y$ be concentric dis- 


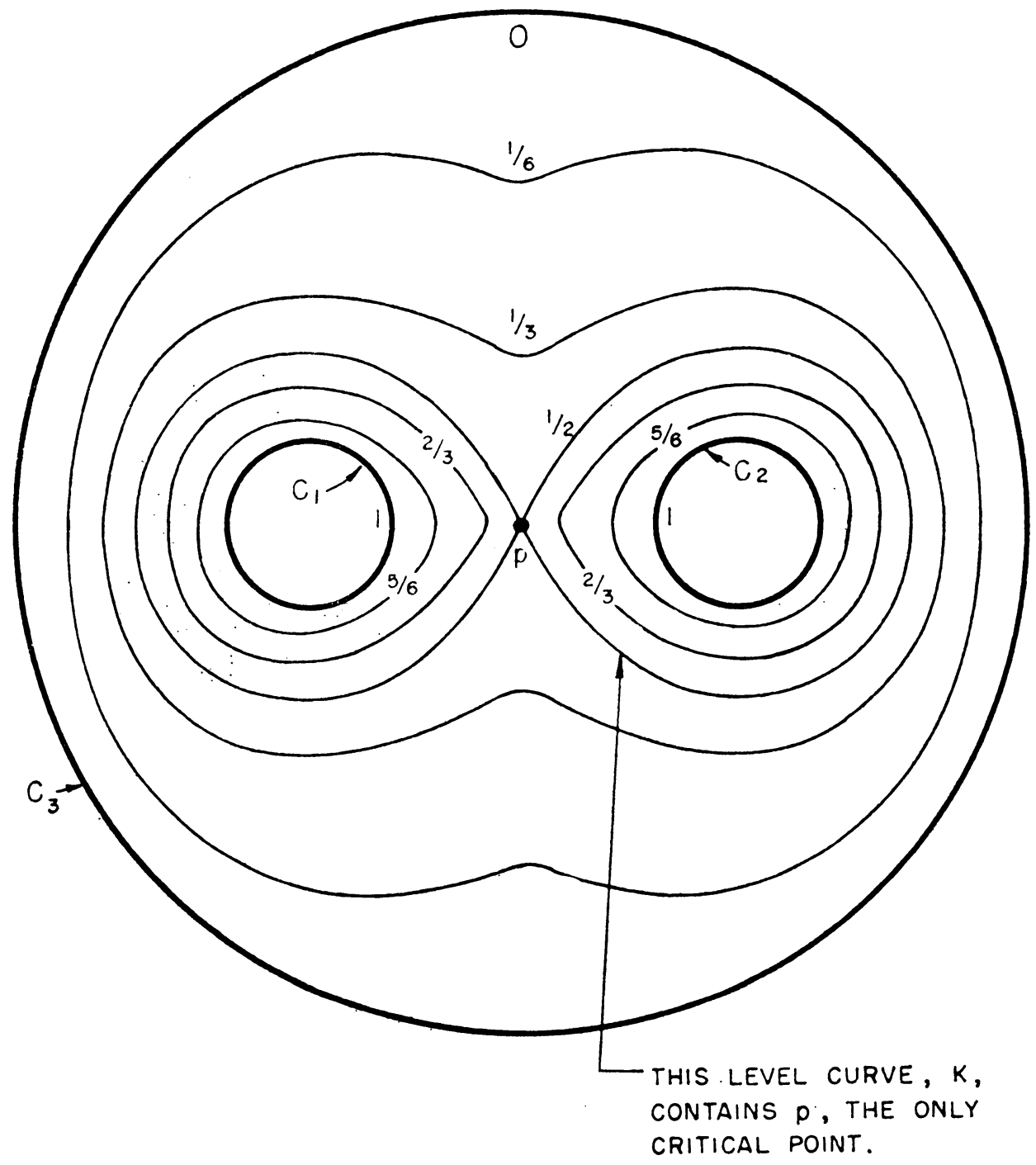

FIG. 1. The level curve structure of a certain pseudo-harmonic function.

joint spirals (starting at $x_{1}$ and $y_{1}$ respectively) each with $p$ as endpoint (see Figure 2), and let $Z$ be the arc of $K$ between $x_{1}$ and $y_{1}$. Let the "rate" with which the spirals $X$ and $Y$ approach the point $p$ be determined by the condition that, for each $n$, there be a homeomorphism $h_{n}$ of the arc $Y_{n}$, in $Y$, between $y_{n}$ and $y_{n+1}$, onto the arc $X_{n+2}$ in $X$, between $x_{n+2}$ and $x_{n+3}$, such that

$$
\left|f(q)-\frac{1}{2}\right|>\left|f\left(h_{n}(q)\right)-\frac{1}{2}\right|
$$




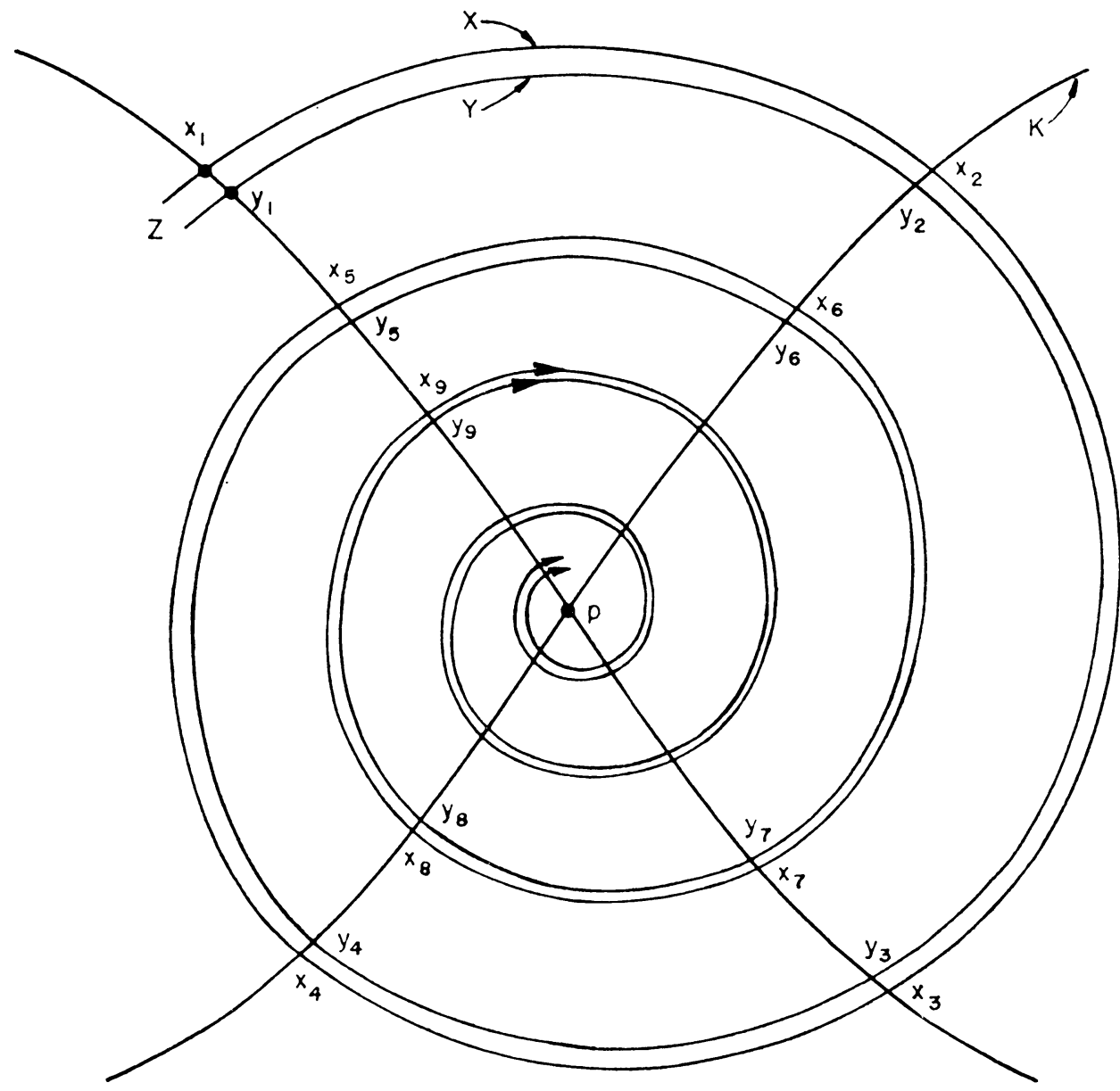

FIG. 2. A domain $M$ spiraling down to the critical point $p$.

for each $q$ in $Y_{n}$ and such that

$$
h_{n}\left(y_{i}\right)=x_{i+2}
$$

for $i=n$ and $n+1$. The purpose of this condition is to assure one that the spirals do not wobble so badly as to spoil the pictures used below. More to the point, let the spirals be chosen in conformity with those pictures!

Let the closure of the domain bounded by $X \cup Y \cup Z$ be denoted by $M$. The function $f: A \rightarrow E^{1}$ is one pseudo-harmonic extension of $f: M \rightarrow E^{1}$ in which the boundary point $p$ of $M$ appears as a critical point. Another $p s e u d o-$ harmonic extension will now be constructed in which $p$ appears as a point of order two, i.e., of multiplicity zero.

Let the domain $M$ be represented as a triangle, as in Figure 3. The func- 


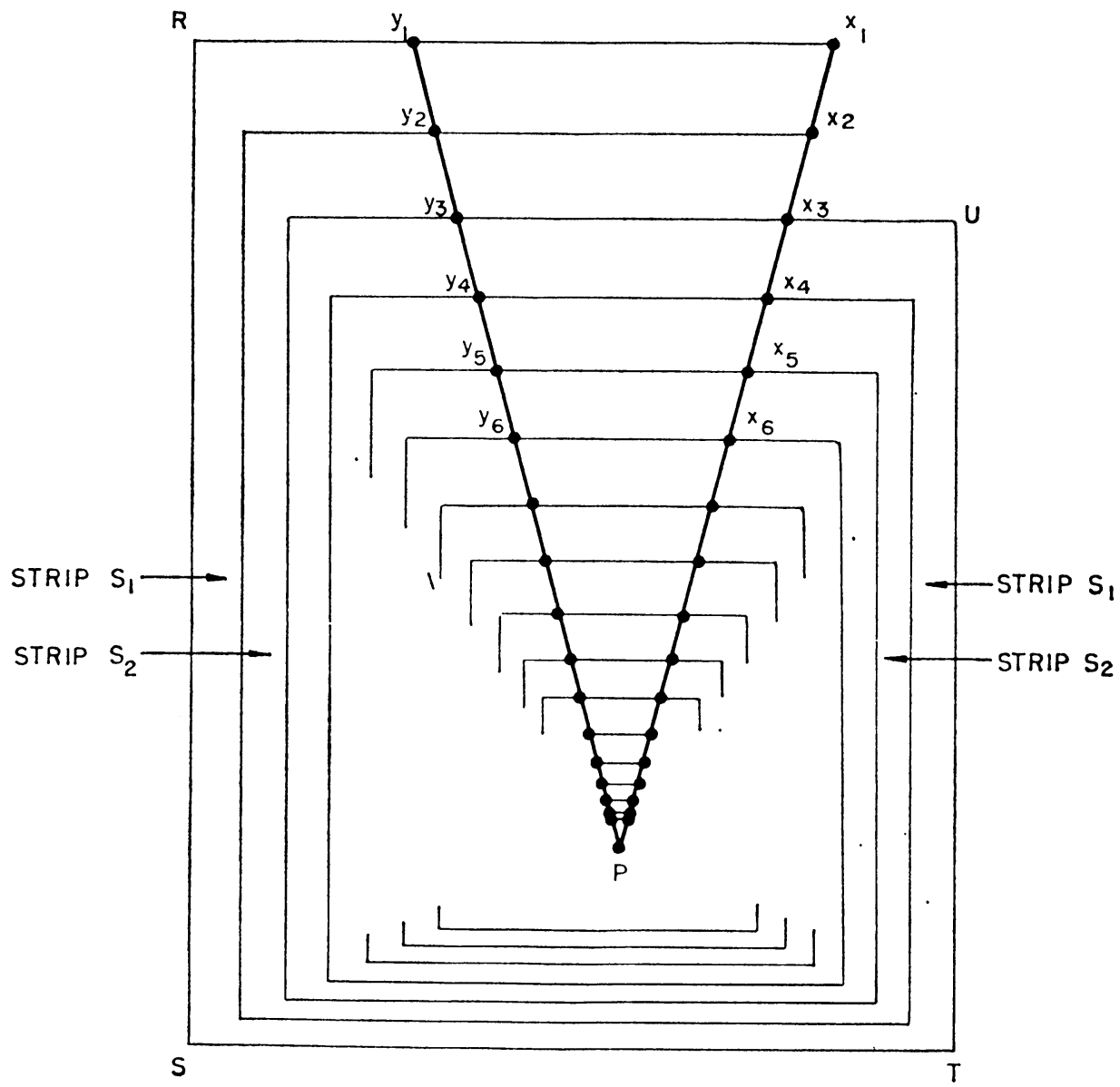

FIG. 3. The extension from $M$ to $D$.

tion $f: M \rightarrow E^{1}$ will be extended to the domain $D$ containing $M$ and bounded by the polygon whose vertices are $R, S, T, U, x_{3}$, and $x_{1}$. The domain $D-M$ has been formed by the union of nonoverlapping bent rectangular strips $S_{n}$, where the strip $S_{n}$ joins the arc $Y_{n}$ in the boundary of $M$ to the $\operatorname{arc} X_{n+2}$ in the boundary of $M$.

The horizontal segments between the points $x_{n}$ and $y_{n}$ in Figure 3 are all $\operatorname{arcs}$ in $K$ and so they bear the $f$-value of $1 / 2$. Similarly the arcs in the boundary of $S_{n}$ from $y_{n}$ to $x_{n+2}$ and from $y_{n+1}$ to $x_{n+3}$ will also bear the $f$-value $1 / 2$. The function $f$ has therefore been given continuous boundary values on the strip $S_{n}$ and is extended to $S_{n}$ as that harmonic function which shares these boundary values. However, this does not make it obvious that the function $f: D \rightarrow E^{1}$ so defined is pseudo-harmonic. A somewhat different description of the extension can be given which makes this property more evident. 


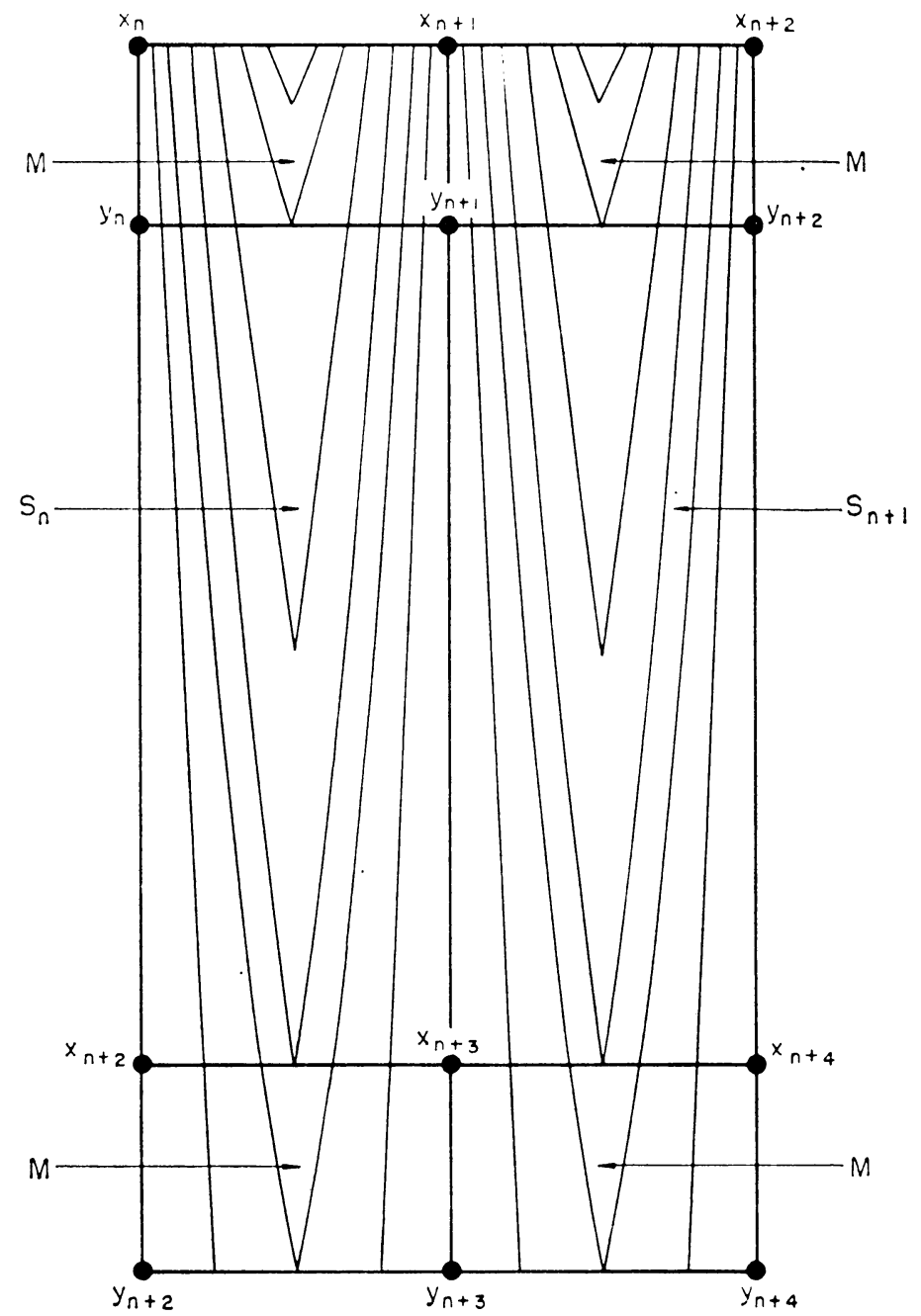

FIG. 4. The extension from $M$ to $S_{n}$ and $S_{n+1}$.

Consider two adjacent strips $S_{n}$ and $S_{n+1}$ drawn vertically with the bends removed and with appropriate portions of the domain $M$ attached at top and bottom as in Figure 4. Suppose $n$ is even so that $f$ is greater than $1 / 2$ on $Y_{n}$ and $X_{n+2}$ and is less than $1 / 2$ on $Y_{n+1}$ and $X_{n+3}$. Then, by condition (1),

$$
f(q)>f\left(h_{n}(q)\right) \geqq \frac{1}{2} \text { for } q \text { in } Y_{n}
$$




$$
\frac{1}{2} \geqq f\left(h_{n+1}(q)\right)>f(q) \text { for } q \text { in } Y_{n+1} \text {. }
$$

Therefore points of $Y_{n}$ can be joined by arcs to the corresponding points in $X_{n+2}$ which bear the same $f$-values, yielding the level curve pattern in $S_{n}$ indicated in Figure 4; a similar construction applies to $S_{n+1}$. The fact that the level curve family of $f$ in $S_{n}$ and $S_{n+1}$ can be drawn as shown in Figure 4 means that $f$ is pseudo-harmonic on $S_{n} \cup S_{n+1}$, in view of Tôki's characterization [3] of pseudo-harmonic functions. Because $f$ is interior on the interior of $D$, and satisfies Tôki's uniform local connectedness condition at each point of $D$ except possibly at $p$, it also follows that $f$ is pseudo-harmonic on $D$.

In this extension $f: D \rightarrow E^{1}$ of $f: M \rightarrow E^{1}$, the level curve containing $p$ is merely an arc with endpoints $x_{1}$ and $x_{2}$, as shown in Figure 3. Thus $p$ is not a critical point of $f: D \rightarrow E^{1}$, although it is a critical point of $f: A \rightarrow E^{1}$.

\section{BiBLIOGRAPHY}

1. William C. Fox, The critical points of Peano-interior functions defined on 2-manifolds, Trans. Amer. Math. Soc. vol. 83 (1956) pp. 338-370.

2. Marston Morse, Topological methods in the theory of functions of a complex variable, Princeton, Princeton University Press, 1947.

3. Yukanari Tôki, A topological characterization of pseudo-harmonic functions, Osaka Math. J. vol. 3 (1951) pp. 101.

4. Yukanari Tôki, and Kôichi Tarumoto, On the pseudo-harmonic functions, Osaka Math. J. vol. 7 (1955) pp. 103-107.

5. W. Kaplan, Mathematical Reviews, 17, 1196.

UNIVERSITY OF MichigAN, AnN Arbor, Mich.

Massachusetts Institute of Technology, Cambridge, Mass. 